\title{
A generalization of Hegedüs-Szilágyi's fixed point theorem in complete metric spaces
}

\section{Tomonari Suzuki*}

\section{"Correspondence:}

suzuki-t@mns.kyutech.ac.jp Department of Basic Sciences, Faculty of Engineering, Kyushu Institute of Technology, Tobata Kitakyushu, 804-8550, Japan

\section{照 Springer}

\begin{abstract}
In 1980, Hegedüs and Szilágyi proved some fixed point theorem in complete metric spaces. Introducing a new contractive condition, we generalize Hegedüs-Szilágyi's fixed point theorem. We discuss the relationship between the new contractive condition and other contractive conditions. We also show that we cannot extend Hegedüs-Szilágyi's fixed point theorem to Meir-Keeler type.
\end{abstract}

MSC: $54 \mathrm{H} 25$

Keywords: Hegedüs-Szilágyi's fixed point theorem; complete metric space

\section{Introduction and preliminaries}

Throughout this paper we denote by $\mathbb{N}$ the set of all positive integers and by $\mathbb{R}$ the set of all real numbers.

Let $T$ be a mapping on a metric space $(X, d)$. Throughout this paper, we define $D_{T}(x)$ and $D_{T}(x, y)$ by

$$
\begin{aligned}
& D_{T}(x)=\sup \left\{d(u, v): u, v \in\left\{x, T x, T^{2} x, \ldots\right\}\right\}, \\
& D_{T}(x, y)=\sup \left\{d(u, v): u, v \in\left\{x, T x, T^{2} x, \ldots, y, T y, T^{2} y, \ldots\right\}\right\}
\end{aligned}
$$

for any $x, y \in X$. That is, $D_{T}(x)$ is the diameter of the orbit $\left\{x, T x, T^{2} x, \ldots\right\}$ of $x$.

Hegedüs and Szilágyi in [1] proved the following fixed point theorem. The author thinks that the proof in [1] is splendid.

Theorem 1 (Theorem 5 in [1]) Let $(X, d)$ be a complete metric space, and let $T$ be a mapping on $X$. Assume $D_{T}(x)<\infty$ for all $x \in X$. Assume also that there exists a function $\varphi$ from $[0, \infty)$ into itself satisfying the following:

(i) $\varphi(t)<t$ holds for all $t \in(0, \infty)$;

(ii) $\varphi$ is upper semicontinuous from the right;

(iii) $d(T x, T y) \leq \varphi \circ D_{T}(x, y)$ holds for all $x, y \in X$.

Then $T$ has a unique fixed point $z$. Moreover, $\left\{T^{n} x\right\}$ converges to $z$ for any $x \in X$.

Remark 1 See also [2-4]. Note that in the proof of Theorem 1 in [3], we need an additional assumption such as the nondecreasingness of $\varphi$.

(c) The Author(s) 2018. This article is distributed under the terms of the Creative Commons Attribution 4.0 International License (http://creativecommons.org/licenses/by/4.0/), which permits unrestricted use, distribution, and reproduction in any medium, provided you give appropriate credit to the original author(s) and the source, provide a link to the Creative Commons license, and indicate if changes were made. 
We state Boyd-Wong's [5], Meir-Keeler's [6] and Matkowski's [7] fixed point theorems.

Theorem 2 (Theorem 1 in [5]) Let $(X, d)$ be a complete metric space, and let $T$ be a mapping on $X$. Assume that there exists a function $\varphi$ from $[0, \infty)$ into itself satisfying (i) and (ii) of Theorem 1 and the following:

(iii) $d(T x, T y) \leq \varphi \circ d(x, y)$ holds for all $x, y \in X$.

Then $T$ has a unique fixed point $z$. Moreover, $\left\{T^{n} x\right\}$ converges to $z$ for any $x \in X$.

Theorem 3 ([6]) Let $(X, d)$ be a complete metric space, and let $T$ be a mapping on $X$. Assume that, for any $\varepsilon>0$, there exists $\delta>0$ such that

$$
d(x, y)<\varepsilon+\delta \quad \text { implies } \quad d(T x, T y)<\varepsilon
$$

for all $x, y \in X$. Then $T$ has a unique fixed point $z$. Moreover, $\left\{T^{n} x\right\}$ converges to $z$ for any $x \in X$.

Theorem 4 (Theorem 1.2 in [7]) Let $(X, d)$ be a complete metric space, and let $T$ be a mapping on $X$. Assume that there exists a function $\varphi$ from $[0, \infty)$ into itself satisfying the following:

(i) $\varphi$ is nondecreasing;

(ii) $\lim _{n} \varphi^{n}(t)=0$ holds for all $t \in(0, \infty)$;

(iii) $d(T x, T y) \leq \varphi \circ d(x, y)$ holds for all $x, y \in X$.

Then $T$ has a unique fixed point $z$. Moreover, $\left\{T^{n} x\right\}$ converges to $z$ for any $x \in X$.

From the above, we can tell that Theorem 1 is of Boyd-Wong [5] type (see Definition 8). So it is a very natural question of whether we can extend Theorem 1 to Meir-Keeler [6] type. It is also a natural question of whether we can prove a Matkowski [7] type fixed point theorem.

In this paper, we answer the above two questions; one is negative and the other is affirmative. Indeed, we generalize Theorem 1. The assumption of the new theorem (Theorem 5) is weaker than a Matkowski type condition (see Corollary 7). We also give a counterexample for a Meir-Keeler type condition (Example 16). We further discuss the relationship between the assumption of Theorem 5 and other contractive conditions.

\section{Main results}

In this section, we generalize Theorem 1.

Theorem 5 Let $(X, d)$ be a complete metric space, and let $T$ be a mapping on $X$. Assume $D_{T}(x)<\infty$ for all $x \in X$. Assume also that there exists a function $\varphi$ from $[0, \infty)$ into itself satisfying the following:

(i) $\varphi(t)<t$ holds for all $t \in(0, \infty)$;

(ii) For any $\varepsilon>0$, there exists $\delta>0$ such that, for any $t \in(0, \infty)$,

$$
\varepsilon<t<\varepsilon+\delta \text { implies } \varphi(t) \leq \varepsilon .
$$

(iii) For any $x, y \in X$,

$$
d(T x, T y) \leq \varphi \circ D_{T}(x, y)
$$


holds.

Then $T$ has a unique fixed point $z$. Moreover, $\left\{T^{n} x\right\}$ converges to $z$ for any $x \in X$.

\section{Remark 2}

- $D_{T}(x, y)<\infty$ obviously holds for any $x, y \in X$.

- Since $D_{T}(x, y)=0$ implies $d(T x, T y)=0$, without loss of generality, we may assume $\varphi(0)=0$.

- We do not assume that $\varphi$ is nondecreasing. So, in general, $D_{T}(T x, T y) \leq \varphi \circ D_{T}(x, y)$ does not hold.

Before proving Theorem 5, we need one lemma.

Lemma 6 Let $x, y \in X$. Assume that either of the following holds:

(a) $x=y$;

(b) $\lim _{n} D_{T}\left(T^{n} x\right)=\lim _{n} D_{T}\left(T^{n} y\right)=0$.

Then $\lim _{n} D_{T}\left(T^{n} x, T^{n} y\right)=0$ holds.

Proof Since

$$
\left\{T^{n} x, T^{n+1} x, \ldots, T^{n} y, T^{n+1} y, \ldots\right\} \supset\left\{T^{n+1} x, T^{n+2} x, \ldots, T^{n+1} y, T^{n+2} y, \ldots\right\}
$$

for $n \in \mathbb{N},\left\{D_{T}\left(T^{n} x, T^{n} y\right)\right\}$ is nonincreasing. So $\left\{D_{T}\left(T^{n} x, T^{n} y\right)\right\}$ converges to some $\varepsilon \in$ $[0, \infty)$. Arguing by contradiction, we assume $\varepsilon>0$. We consider the following two cases:

- $\varepsilon<D_{T}\left(T^{n} x, T^{n} y\right)$ holds for any $n \in \mathbb{N}$;

- $\varepsilon=D_{T}\left(T^{n} x, T^{n} y\right)$ holds for some $n \in \mathbb{N}$.

In the first case, we choose $\delta \in(0, \infty)$ such that

$$
\varepsilon<t<\varepsilon+\delta \quad \text { implies } \quad \varphi(t) \leq \varepsilon .
$$

We choose $v \in \mathbb{N}$ satisfying

$$
D_{T}\left(T^{v} x, T^{v} y\right)<\varepsilon+\delta .
$$

In the case of (b), without loss of generality, we may assume

$$
D_{T}\left(T^{v} x\right) \leq \varepsilon / 2 \quad \text { and } \quad D_{T}\left(T^{v} y\right) \leq \varepsilon / 2 .
$$

Fix $m \geq v$ and $n \geq v$. Then since

$$
\begin{aligned}
\varepsilon & <D_{T}\left(T^{\max \{m, n\}} x, T^{\max \{m, n\}} y\right) \\
& \leq D_{T}\left(T^{m} x, T^{n} y\right) \\
& \leq D_{T}\left(T^{\min \{m, n\}} x, T^{\min \{m, n\}} y\right) \leq D_{T}\left(T^{v} x, T^{v} y\right)<\varepsilon+\delta,
\end{aligned}
$$

we have

$$
d\left(T^{m+1} x, T^{n+1} y\right) \leq \varphi \circ D_{T}\left(T^{m} x, T^{n} y\right) \leq \varepsilon .
$$


Since $m, n$ are arbitrary, considering (1), we obtain

$$
\varepsilon<D_{T}\left(T^{v+1} x, T^{\nu+1} y\right) \leq \varepsilon,
$$

which implies a contradiction. In the second case, we choose $v \in \mathbb{N}$ satisfying

$$
D_{T}\left(T^{v} x, T^{v} y\right)=\varepsilon .
$$

In the case of (b), without loss of generality, we may assume

$$
D_{T}\left(T^{v} x\right) \leq \varphi(\varepsilon) \quad \text { and } \quad D_{T}\left(T^{v} y\right) \leq \varphi(\varepsilon) .
$$

Fix $m \geq v$ and $n \geq v$. Then since

$$
\begin{aligned}
\varepsilon & \leq D_{T}\left(T^{\max \{m, n\}} x, T^{\max \{m, n\}} y\right) \\
& \leq D_{T}\left(T^{m} x, T^{n} y\right) \\
& \leq D_{T}\left(T^{\min \{m, n\}} x, T^{\min \{m, n\}} y\right) \leq D_{T}\left(T^{v} x, T^{v} y\right)=\varepsilon,
\end{aligned}
$$

we have

$$
d\left(T^{m+1} x, T^{n+1} y\right) \leq \varphi \circ D_{T}\left(T^{m} x, T^{n} y\right)=\varphi(\varepsilon) .
$$

Since $m, n$ are arbitrary, considering (2), we obtain

$$
\varepsilon \leq D_{T}\left(T^{v+1} x, T^{v+1} y\right) \leq \varphi(\varepsilon)<\varepsilon
$$

which implies a contradiction. Therefore we have shown $\lim _{n} D_{T}\left(T^{n} x, T^{n} y\right)=0$.

Proof of Theorem 1 Fix $x \in X$. By Lemma 6(a), $\left\{D_{T}\left(T^{n} x\right)\right\}$ converges to 0 . Thus $\left\{T^{n} x\right\}$ is a Cauchy sequence in $X$. Since $X$ is complete, $\left\{T^{n} x\right\}$ converges to some $z \in X$. By Lemma 6(a) again, $\left\{D_{T}\left(T^{n} z\right)\right\}$ also converges to 0 . So, by Lemma $6(\mathrm{~b})$, we obtain

$$
\lim _{n \rightarrow \infty} D_{T}\left(T^{n} x, T^{n} z\right)=0 .
$$

So $\left\{T^{n} z\right\}$ also converges to $z$. Hence

$$
D_{T}(z)=D_{T}(T z)
$$

holds. Arguing by contradiction, we assume $\varepsilon:=D_{T}(z)>0$. Since $\lim _{n} D_{T}\left(T^{n} z\right)=0$ holds, there exists $v \in \mathbb{N}$ satisfying

$$
\varepsilon=D_{T}(z)=\cdots=D_{T}\left(T^{v-1} z\right)=D_{T}\left(T^{v} z\right)>D_{T}\left(T^{v+1} z\right),
$$

where $T^{0} z=z$. This implies

$$
\varepsilon=D_{T}\left(T^{v} z\right)=\sup \left\{d\left(T^{v} z, T^{n} z\right): n>v\right\} .
$$


For $n>v$, we have

$$
d\left(T^{\nu} z, T^{n} z\right) \leq \varphi \circ D_{T}\left(T^{\nu-1} z, T^{n-1} z\right)=\varphi \circ D_{T}\left(T^{\nu-1} z\right)=\varphi(\varepsilon) .
$$

Since $n$ is arbitrary, we obtain

$$
\varepsilon=\sup \left\{d\left(T^{v} z, T^{n} z\right): n>v\right\} \leq \varphi(\varepsilon)<\varepsilon,
$$

which implies a contradiction. Therefore we have shown $D_{T}(z)=0$. Hence $z$ is a fixed point of $T$. Since (3) holds for any $x \in X$, we obtain the uniqueness of the fixed point.

By Theorem 5, we obtain a Matkowski type fixed point theorem.

Corollary 7 Let $(X, d)$ be a complete metric space, and let $T$ be a mapping on $X$. Assume $D_{T}(x)<\infty$ for all $x \in X$. Assume also that there exists a function $\varphi$ from $[0, \infty)$ into itself satisfying the following:

(i) $\varphi$ is nondecreasing;

(ii) $\lim _{n} \varphi^{n}(t)=0$ holds for all $t \in(0, \infty)$;

(iii) $d(T x, T y) \leq \varphi \circ D_{T}(x, y)$ holds for all $x, y \in X$.

Then $T$ has a unique fixed point $z$. Moreover, $\left\{T^{n} x\right\}$ converges to $z$ for any $x \in X$.

\section{Comparison}

In this section, using subsets of $(0, \infty)^{2}$, we discuss the relationship between the new contractive condition in Theorem 5 and other contractive conditions. See $[1,8-11]$ and the references therein.

Definition 8 Let $Q$ be a subset of $(0, \infty)^{2}$.

(1) $Q$ is said to be contractive (Cont for short) [12,13] if there exists $r \in(0,1)$ such that $u \leq r t$ holds for any $(t, u) \in Q$.

(2) $Q$ is said to be Browder (Bro for short) [14] if there exists a function $\varphi$ from $(0, \infty)$ into itself satisfying the following:

(2-i) $\varphi$ is nondecreasing and right-continuous;

(2-ii) $\varphi(t)<t$ holds for any $t \in(0, \infty)$;

(2-iii) $u \leq \varphi(t)$ holds for any $(t, u) \in Q$.

(3) $Q$ is said to be Boyd-Wong (BW for short) [5] if there exists a function $\varphi$ from $(0, \infty)$ into itself satisfying the following:

(3-i) $\varphi$ is upper semicontinuous from the right;

(3-ii) $\varphi(t)<t$ holds for any $t \in(0, \infty)$;

(3-iii) $u \leq \varphi(t)$ holds for any $(t, u) \in Q$.

(4) $Q$ is said to be Meir-Keeler (MK for short) [6] if, for any $\varepsilon>0$, there exists $\delta>0$ such that $u<\varepsilon$ holds for any $(t, u) \in Q$ with $t<\varepsilon+\delta$.

(5) $Q$ is said to be Matkowski (Mat for short) [7] if there exists a function $\varphi$ from $(0, \infty)$ into itself satisfying the following:

(5-i) $\varphi$ is nondecreasing;

(5-ii) $\lim _{n} \varphi^{n}(t)=0$ for any $t \in(0, \infty)$;

(5-iii) $u \leq \varphi(t)$ holds for any $(t, u) \in Q$. 
(6) $Q$ is said to be of New-type (NT for short) if there exists a function $\varphi$ from $(0, \infty)$ into itself satisfying the following:

(6-i) $\varphi(t)<t$ for any $t \in(0, \infty)$;

(6-ii) For any $\varepsilon>0$, there exists $\delta>0$ such that $\varepsilon<t<\varepsilon+\delta$ implies $\varphi(t) \leq \varepsilon$;

(6-iii) $u \leq \varphi(t)$ holds for any $(t, u) \in Q$.

(7) $Q$ is said to be $C J M[15-18]$ if the following hold:

(7-i) For any $\varepsilon>0$, there exists $\delta>0$ satisfying $u \leq \varepsilon$ holds for any $(t, u) \in Q$ with $t<\varepsilon+\delta$;

(7-ii) $u<t$ holds for any $(t, u) \in Q$.

It is obvious that the following implications hold:

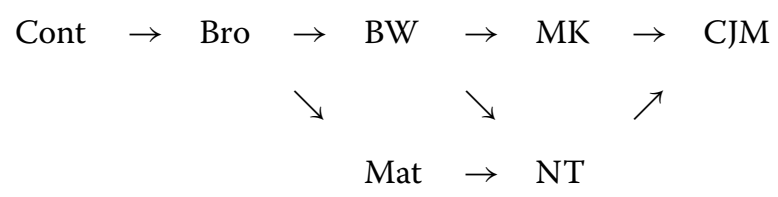

It is well known that the converse implication of (Cont $\rightarrow$ Bro) does not hold. The following three examples tell us that for each implication except (Cont $\rightarrow$ Bro), there exists a counterexample for its converse implication. In particular, MK and NT are independent.

Example 9 Let $u \in(0, \infty)$ and define $Q$ by

$$
Q=\{(t, u): u<t\}
$$

Then $Q$ is Mat. However, $Q$ is not MK.

Remark 3 We note that the converse implication of $(\mathrm{BW} \rightarrow$ NT) does not hold.

Example 10 Let $t, u \in(0, \infty)$ with $t<u$. Define $Q$ by

$$
Q=\{((1-\lambda) t+\lambda u, \lambda u): \lambda \in(0,1)\} .
$$

Then $Q$ is $B W$. However, $Q$ is not Mat.

Remark 4 We note that the converse implication of $(\mathrm{Mat} \rightarrow \mathrm{NT})$ does not hold.

Example 11 Let $t \in(0, \infty)$ and define $Q$ by

$$
Q=\{(t, u): 0<u<t\} .
$$

Then $Q$ is MK. However, $Q$ is not NT.

Remark 5 We note that the converse implication of $(\mathrm{NT} \rightarrow \mathrm{CJM})$ does not hold.

In the remainder of this section, we let $(X, d)$ be a complete metric space, and let $T$ be a mapping on $X$ satisfying $D_{T}(x)<\infty$ for all $x \in X$. Define subsets $P_{T}$ and $Q_{T}$ of $(0, \infty)^{2}$ 
by

$$
\begin{aligned}
& P_{T}=\{(d(x, y), d(T x, T y)): x, y \in X\} \cap(0, \infty)^{2}, \\
& Q_{T}=\left\{\left(D_{T}(x, y), d(T x, T y)\right): x, y \in X\right\} \cap(0, \infty)^{2} .
\end{aligned}
$$

We will give three mappings such that $Q_{T}$ for each mapping matches one of Examples 9-11, respectively.

Lemma 12 Let $X$ be a nonempty set. Let $f$ be a function from $X$ into $[0, \infty)$ such that $\{x \in X: f(x)=0\}$ consists of at most one element. Define a function $d$ from $X \times X$ into $[0, \infty)$ by

$$
d(x, y)= \begin{cases}0 & \text { if } x=y \\ \max \{f(x), f(y)\} & \text { if } x \neq y\end{cases}
$$

Let $T$ be a mapping on $X$ satisfying the following:

- $f(x)>0$ implies $T x \neq x$ and $f(T x) \leq f(x)$

- $f(x)=0$ implies $T x=x$.

Then the following hold:

(i) $(X, d)$ is a metric space;

(ii) if either $\{x \in X: f(x)=0\} \neq \varnothing$ or $\inf f(X)>0$ holds, then $X$ is complete;

(iii) $P_{T}=Q_{T}$.

Proof We have essentially proved (i) and (ii); see Lemma 7 in [19]. Let us prove (iii). Fix $x, y \in X$ with $x \neq y$ and $f(x) \leq f(y)$. Then we have $f(y)>0$ and hence $T y \neq y$. We have

$$
\begin{aligned}
& \cdots \leq f\left(T^{n} y\right) \leq \cdots \leq f\left(T^{2} y\right) \leq f(T y) \leq f(y), \\
& \cdots \leq f\left(T^{n} x\right) \leq \cdots \leq f\left(T^{2} x\right) \leq f(T x) \leq f(x) \leq f(y) .
\end{aligned}
$$

Hence

$$
D_{T}(x, y)=f(y)=d(x, y)
$$

holds. Therefore $P_{T}=Q_{T}$ holds.

Example 13 Let $X=[0, \infty)$ and define a function $d$ from $X \times X$ into $[0, \infty)$ by (5), where $f(x)=x$. That is,

$$
d(x, y)= \begin{cases}0 & \text { if } x=y, \\ \max \{x, y\} & \text { if } x \neq y\end{cases}
$$

holds. Define a mapping $T$ on $X$ by

$$
T x= \begin{cases}0 & \text { if } x \leq 1, \\ 1 & \text { if } x>1\end{cases}
$$

Then the following hold: 
(i) $(X, d)$ is a complete metric space;

(ii) $f(x)>0$ implies $f(T x)<f(x)$;

(iii) $f(x)=0$ implies $T x=x$;

(iv) $P_{T}=Q_{T}=\{(t, 1): 1<t\}$;

(v) $P_{T}$ and $Q_{T}$ are Mat;

(vi) neither $P_{T}$ nor $Q_{T}$ are $M K$.

Proof We can prove (i)-(iii) easily. Using Lemma 12, we can prove (iv). (v) and (vi) follow from Example 9.

Example 14 Put $X=[0,2)$ and define $f$ and $d$ as in Example 13. Define a mapping $T$ on $X$ by

$$
T x= \begin{cases}0 & \text { if } x \leq 1, \\ 2 x-2 & \text { if } x \geq 1\end{cases}
$$

Then (i)-(iii) of Example 13 and the following hold:

(iv) $P_{T}=Q_{T}=\{(1+\lambda, 2 \lambda): \lambda \in(0,1)\}$;

(v) $P_{T}$ and $Q_{T}$ are BW;

(vi) neither $P_{T}$ nor $Q_{T}$ are Mat.

Proof We can prove (i)-(iii) easily. Using Lemma 12, we can prove (iv). (v) and (vi) follow from Example 10.

Example 15 Let $X=[0,1) \cup(1, \infty)$ and define a function $d$ from $X \times X$ into $[0, \infty)$ by (5), where $f(x)=\min \{x, 1\}$. Define a mapping $T$ on $X$ by

$$
T x= \begin{cases}0 & \text { if } x<1 \\ 1 / x & \text { if } x>1\end{cases}
$$

Then (i)-(iii) of Example 13 and the following hold:

(iv) $P_{T}=Q_{T}=\{(1, u): 0<u<1\}$;

(v) $P_{T}$ and $Q_{T}$ are $M K$;

(vi) neither $P_{T}$ nor $Q_{T}$ are NT.

Proof We can prove (i)-(iii) easily. Using Lemma 12, we can prove (iv). (v) and (vi) follow from Example 11.

We finally give the following example, which tells us that we cannot extend Theorem 1 to a Meir-Keeler type contractive condition.

Example 16 Let $X=[0,1)$ and define a function $d$ from $X \times X$ into $[0, \infty)$ by (6). Define a mapping $T$ on $X$ by

$$
T x= \begin{cases}1 / 2 & \text { if } x=0, \\ \sqrt{x} & \text { if } x \neq 0 .\end{cases}
$$


Then the following hold:

(i) $(X, d)$ is a complete metric space;

(ii) $d(x, y)<1$ holds for any $x, y \in X$;

(iii) for any $x \in X,\left\{T^{n} x\right\}$ converges to 1 in the Euclidean space $\mathbb{R}^{1}$;

(iv) $D_{T}(x)=1$ holds for any $x \in X$;

(v) $T X=(0,1)$;

(vi) $Q_{T}=\{(1, u): 0<u<1\}$;

(vii) $Q_{T}$ is $M K$;

(viii) $Q_{T}$ is not NT;

(ix) $T$ does not have a fixed point.

Proof We can easily prove (i)-(vi) and (ix). (vii) and (viii) follow from Example 11.

\section{Conclusions}

In this paper, introducing a new contractive condition (see Definition 8(6)), we generalize Hegedüs-Szilágyi's fixed point theorem (Theorem 1) in complete metric spaces proved in 1980. In Section 3, we discuss the relationship between the new contractive condition and other contractive conditions. We also show that we cannot extend Theorem 1 to MeirKeeler type (see Example 16).

Acknowledgements

The author is supported in part by JSPS KAKENHI Grant Number 16K05207 from Japan Society for the Promotion of Science.

\section{Competing interests}

The author declares that he has no competing interests.

Authors' contributions

The author read and approved the final manuscript.

\section{Publisher's Note}

Springer Nature remains neutral with regard to jurisdictional claims in published maps and institutional affiliations.

Received: 7 July 2017 Accepted: 12 December 2017 Published online: 08 January 2018

\section{References}

1. Hegedüs, M, Szilágyi, T: Equivalent conditions and a new fixed point theorem in the theory of contractive type mappings. Math. Jpn. 25, 147-157 (1980)

2. Hegedüs, M: New generalizations of Banach's contraction principle. Acta Sci. Math. 42, 87-89 (1980)

3. Tasković, MR: Some results in the fixed point theory, II. Publ. Inst. Math. (Belgr.) 27, 249-258 (1980)

4. Walter, W: Remarks on a paper by F. Browder about contraction. Nonlinear Anal. 5, 21-25 (1981)

5. Boyd, DW, Wong, JSW: On nonlinear contractions. Proc. Am. Math. Soc. 20, 458-464 (1969)

6. Meir, A, Keeler, E: A theorem on contraction mappings. J. Math. Anal. Appl. 28, 326-329 (1969)

7. Matkowski, J: Integrable Solutions of Functional Equations. Diss. Math., vol. 127. Institute of Mathematics Polish Academy of Sciences, Warsaw (1975)

8. Jachymski, J: Remarks on contractive conditions of integral type. Nonlinear Anal. 71, 1073-1081 (2009)

9. Meszáros, J: A comparison of various definitions of contractive type mappings. Bull. Calcutta Math. Soc. 84, 167-194 (1992)

10. Rhoades, BE: A comparison of various definitions of contractive mappings. Trans. Am. Math. Soc. 226, $257-290$ (1977)

11. Suzuki, T: Discussion of several contractions by Jachymski's approach. Fixed Point Theory Appl. 2016, Article ID 91 (2016)

12. Banach, S: Sur les opérations dans les ensembles abstraits et leur application aux équations intégrales. Fundam. Math. 3, 133-181 (1922)

13. Caccioppoli, R: Un teorema generale sull'esistenza di elementi uniti in una transformazione funzionale. Rend. Accad. Naz. Lincei 11, 794-799 (1930)

14. Browder, FE: On the convergence of successive approximations for nonlinear functional equations. Proc. K. Ned. Akad. Wet., Ser. A, Indag. Math. 30, 27-35 (1968)

15. Ćirić, L: A new fixed-point theorem for contractive mappings. Publ. Inst. Math. (Belgr.) 30, 25-27 (1981)

16. Jachymski, J: Equivalent conditions and the Meir-Keeler type theorems. J. Math. Anal. Appl. 194, $293-303$ (1995) 
17. Kuczma, M, Choczewski, B, Ger, R: Iterative Functional Equations. Encyclopedia of Mathematics and Its Applications, vol. 32. Cambridge University Press, Cambridge (1990)

18. Matkowski, J: Fixed point theorems for contractive mappings in metric spaces. Čas. Pěst. Mat. 105, 341-344 (1980)

19. Suzuki, T, Alamri, B: A sufficient and necessary condition for the convergence of the sequence of successive approximations to a unique fixed point II. Fixed Point Theory Appl. 2015, Article ID 59 (2015)

Submit your manuscript to a SpringerOpen ${ }^{\circ}$ journal and benefit from:

- Convenient online submission

$\checkmark$ Rigorous peer review

- Open access: articles freely available online

- High visibility within the field

- Retaining the copyright to your article

Submit your next manuscript at $\boldsymbol{~ s p r i n g e r o p e n . c o m ~}$ 